\title{
The Role of the Lateral Habenula in Inhibitory Learning from Reward Omission
}

\author{
DRodrigo Sosa, Jesús Mata-Luévanos, and Mario Buenrostro-Jáuregui
}

https://doi.org/10.1523/ENEURO.0016-21.2021

${ }^{1}$ Universidad Panamericana, Escuela de Pedagogía, 49 Álvaro del Portillo, Ciudad Granja, Zapopan, 45010, Mexico and

${ }^{2}$ Universidad Iberoamericana, Laboratorio de Neurociencias, 880 Prolongación Paseo de la Reforma, Lomas de Santa Fé, Mexico City, 01219, Mexico

\begin{abstract}
The lateral habenula $(\mathrm{LHb})$ is a phylogenetically primitive brain structure that plays a key role in learning to inhibit distinct responses to specific stimuli. This structure is activated by primary aversive stimuli, cues predicting an imminent aversive event, unexpected reward omissions, and cues associated with the omission of an expected reward. The most widely described physiological effect of LHb activation is acutely suppressing midbrain dopaminergic signaling. However, recent studies have identified multiple means by which the LHb promotes this effect as well as other mechanisms of action. These findings reveal the complex nature of $\mathrm{LHb}$ circuitry. The present paper reviews the role of this structure in learning from reward omission. We approach this topic from the perspective of computational models of behavioral change that account for inhibitory learning to frame key findings. Such findings are drawn from recent behavioral neuroscience studies that use novel brain imaging, stimulation, ablation, and reversible inactivation techniques. Further research and conceptual work are needed to clarify the nature of the mechanisms related to updating motivated behavior in which the $\mathrm{LHb}$ is involved. As yet, there is little understanding of whether such mechanisms are parallel or complementary to the well-known modulatory function of the more recently evolved prefrontal cortex.
\end{abstract}

Key words: dopamine signaling; conditioned inhibition; inhibitory control; mesolimbic pathway; mesocortical pathway; negative reward prediction error

\section{Significance Statement}

The lateral habenula ( $\mathrm{LHb}$ ) is a brain structure that has received a great deal of attention and has been a hot topic in the past decades. Consequently, this research field has been extensively reviewed. We review in detail some key recent findings that are pivotal in framing the role of the LHb in well-described associative learning phenomenon, conditioned inhibition. This specific topic has not been considered deeply enough in previous review articles. We also outline the possible mechanisms by which the LHb updates behavior by means of two identified pathway categories (inhibitory and excitatory). This provides a comprehensive account potentially embracing more issues than previously thought, refines our understanding of multiple reward-related mechanisms, and raises novel research questions.

\section{Introduction}

The lateral habenula $(\mathrm{LHb})$ is a phylogenetically preserved brain structure located in the dorsomedial surface of the thalamus that participates in learning from aversive (i.e., undesired) experiences. These include primary aversive stimuli, reward omission, and cues associated with either aversive

Received January 12, 2021; accepted March 30, 2021; First published May 7, 2021.

The authors declare no competing financial interests. stimuli or reward omission (Baker et al., 2016). However, latest research suggests that the $\mathrm{LHb}$ is not as crucial for learning from primary aversive experiences (see Li et al., 2019) as it is for learning from reward omission.

The LHb has been characterized as a part of a "brake" mechanism to suppress firing in midbrain dopaminergic

Author contributions: R.S., conceived the idea and outlined the paper; R.S., J.M.-L., and M.B.-J. wrote the paper to equal extent; R.S. and M.B.-J. worked out the figures; J.M.-L. formatted the manuscript. 
neurons (Barrot et al., 2012; Vento and Jhou, 2020). Such effect is mainly achieved by exciting GABAergic neurons in the rostromedial tegmental nucleus (RMTg) reaching the ventral tegmental area (VTA) and the substantia nigra pars compacta (SNc; Jhou et al., 2009). However, direct glutamatergic excitation of GABAergic interneurons that synapse with dopamine neurons in the VTA also takes place (Omelchenko and Sesack, 2009). In both cases, the net effect is to suppress dopamine release in the nucleus accumbens (NAc; by VTA endings) and the dorsal striatum (by SNc endings). This would disrupt reward-related motor activity, and reward-related plastic changes associated with midbrain dopamine release (Tsutsui-Kimura et al., 2020). A third, recently discovered, pathway (Lammel et al., 2012) involves LHb glutamatergic projections reaching dopamine neurons in the VTA that, in turn, target the medial prefrontal cortex (mPFC; see Fig. 1). mPFC activity has been associated with aversive learning (Huang et al., 2019) and the capacity to behave congruently with hierarchically arranged stimuli sets (Roughley and Killcross, 2019).

Numerous reviews about the physiology of the LHb have already been published; most of them cover the participation of the LHb in primary aversive learning, stressing on the its putative implications for psychiatric conditions (Hu et al., 2020). Other settings that involve the $\mathrm{LHb}$ in non-primary aversive situations, such as tasks that require behavior flexibility, have also been reviewed (Baker et al., 2016). In this review, we discuss the experimental findings on a third ground, the role of the LHb in reward-omission learning, which is known to imply negative reward prediction errors. Particularly, we explore how such findings could be framed in terms of some models of behavioral change. This might be informative for a research agenda aiming to unveil the functional basis of adaptive mechanisms in which the LHb participates. In addition, this may aid in understanding maladaptive behaviors that arise from the disruption of brain regions in the extended network in which the LHb is embedded. We conceive learning from reward omission as being potentially integrated with more widely studied phenomena involving $\mathrm{LHb}$ function (i.e., primary aversive conditioning and behavioral flexibility). On one hand, reward-omission experiences imbue preceding cues with properties that are functionally equivalent to those cues associated with primary aversive stimuli. On the other hand, learning in tasks requiring behavioral flexibility involves committing

This work was supported by the Sistema Nacional de Investigadores (SNI) grant for RS and MB-J, and by the Consejo Nacional de Ciencia y Tecnología (CONACyT) postdoctoral fellowship grant for J.M.-L.

Acknowledgements: We thank the División de Investigación y Posgrado of the Universidad Iberoamericana Ciudad de México and the Fondo Fomento a la Investigación of the Universidad Panamericana for providing funding for production expenses.

Correspondence should be addressed to Rodrigo Sosa at rsosas@up.edu. $\mathrm{mx}$ or Mario Buenrostro-Jáuregui at mario.buenrostro@ibero.mx.

https://doi.org/10.1523/ENEURO.0016-21.2021

Copyright (C) 2021 Sosa et al.

This is an open-access article distributed under the terms of the Creative Commons Attribution 4.0 International license, which permits unrestricted use, distribution and reproduction in any medium provided that the original work is properly attributed. errors (Baker et al., 2017) and this often entails the omission of otherwise expected rewards. Therefore, we hope that understanding one of these artificially delineated fields would potentially help to elucidate some of the mechanisms involved in the others.

\section{Modeling the Dynamics of Acquisition and Extinction of Responding in Reward Learning}

Learning could be roughly regarded as a process in which behavior is updated when an organism faces environmental regularities. Although learning is a continuous process (Sutton and Barto, 2018), it is sometimes methodologically and analytically useful to discretize it in trials (Harris, 2019). Trials are fractions of time in which explicit experiences are assumed to promote specific changes in future behavior. Rescorla and Wagner (1972) proposed a model accounting for the associative strength of a target stimulus given its pairings with an affectively significant event on a trial-by-trial basis. Such an associative strength or value could represent a theoretical estimation of either the vigour or the probability of responding to the target stimulus in the next trial. While "responding" was originally intended to reflect a change in overt outcomeanticipating actions, it may also represent a neural-level state change such as firing of dopamine neurons (see Roesch et al., 2012). This model states that a target stimulus gains or loses associative strength according to a learning rule. The change for this value in a given trial depends on the discrepancy of the current value of the target stimulus and that of the outcome that follows (e.g., presentation or absence of a reward). Formally:

$$
\Delta V_{X}=\alpha\left(\lambda-\Sigma V_{N}\right),
$$

where $\Delta V_{X}$ represents the change in the associative strength $(V$ ) of the target stimulus (or action; $X$ ) in a given trial, $\lambda$ represents the magnitude of reward (zero if the reward is omitted), $\Sigma V_{N}$ represents the sum of the values of all cues that are present during a conditioning trial (usually, accounted for with a few of those cues), and $\alpha$, which is bound between 0 and 1, represents a learning rate parameter. To show how the model operates, let us first consider the simplest possible example. This requires assuming that, during conditioning trials, the only relevant input besides the reward is a single cue (thus being $V_{x} \approx$ $\left.\Sigma V_{N}\right)$. If $\lambda(>0)$ and $\alpha$ are held constant, $V_{x}$ will increase approaching $\lambda$ throughout conditioning trials (e.g., pairings of the stimulus with reward) in a decelerated fashion, as the discrepancy between these parameters (i.e., $\lambda-\Sigma V_{N}$ ) progressively declines (see Fig. 2, left panel). Thence, the absolute value of the parenthetical term in Equation 1 determines the amount of behavioral change on the next trial of the same type. The discrepancy between expected and experienced outcomes has been termed prediction error and could be regarded as a theoretical teaching signal that alters some aspect of the system to update behavior. Prediction errors can be classified as appetitive (reward-related) or aversive, and as positive or negative (lordanova et al., 2021). In addition, 


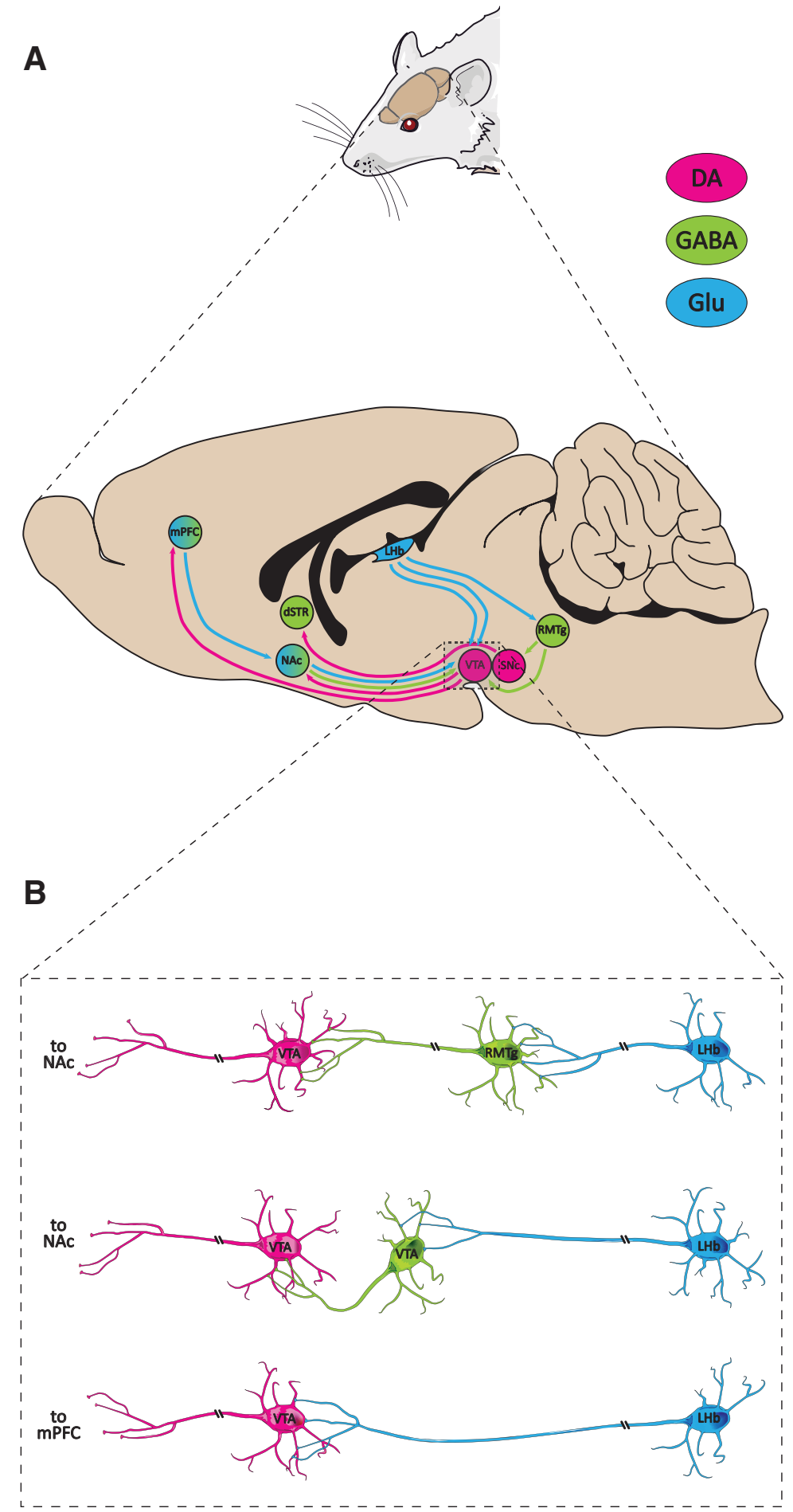

Figure 1. Projections of the midbrain dopamine regions and their neurochemical interaction with the LHb. $\boldsymbol{A}$, Sagittal view of the rat brain, depicting efferent pathways of midbrain dopamine regions and their input from the LHb. $B$, Synaptic relationships between neurons in the VTA and inputs (direct and through the RMTg) from the LHb. DA, dopamine (orange); GABA, green; Glu, glutamate (blue); dSTR, dorsal striatum. The image of the rat was adapted from https://smart.servier.com/smart image/rat, under the terms of the CC-BY 3.0 Unported license (https://creativecommons.org/licenses/by/3.0/). Rat brain adapted from Juarez et al. (2013).

prediction errors seem to dictate both Pavlovian (i.e., stimulus-outcome) and instrumental (i.e., action-outcome) learning through a homologous correction mechanism (Bouton et al., 2020; see also Eder et al., 2015).
A the physiological level, canonical midbrain dopamine neurons fire above their baseline activity upon the presentation of unexpected rewards (i.e., positive reward prediction error; $\lambda>\Sigma V_{N}$ ). Such dopamine bursts 


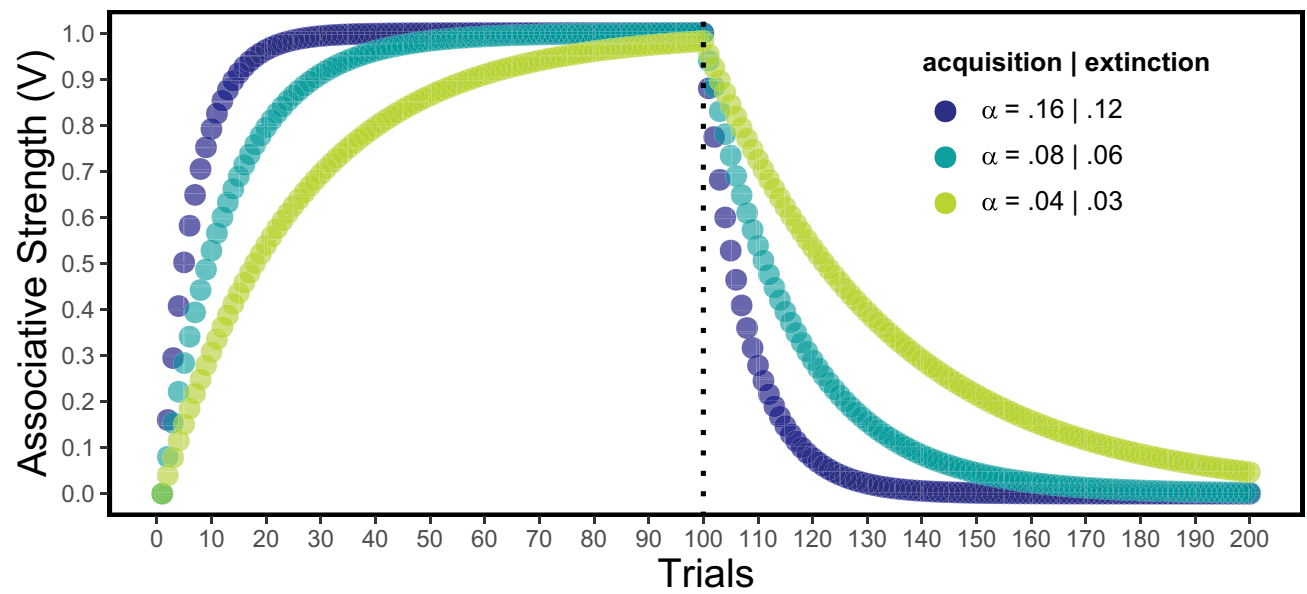

Figure 2. Dynamics of associative strength according to the Rescorla-Wagner model in 100 consecutive trials of acquisition $(\lambda=1)$ followed by 100 extinction trials $(\lambda=0)$. Colors represent different learning rates $(\alpha)$, assuming that extinction rates are $3 / 4$ of acquisition rates. Note that the decrease in responding (right panel) is a dynamic process that progresses with each trial, unlike the slow and continuous decay that would be expected if we simply allow time to pass.

increase the probability of occurrence of the actions that took place priorly, whenever the organism is presented to a similar situation in the future (i.e., positive reinforcement). In addition, dopamine bursts backpropagate to reliable signals of reward, making them acquire signaling (i.e., discrimination learning) and rewarding (i.e., conditioned reinforcement) properties in themselves (Sutton and Barto, 2018). Dopamine phasic activity (as in $V_{X}>0$ ) is triggered by inputs received by midbrain neurons from multiple nodes in a brain-wide network subsequent to sensory processing (Tian et al., 2016). Deceleration in the increase of associative strength with repeated experiences involving paired presentations of the stimulus and the reward would require an antagonistic mechanism. Inhibitory NAc-VTA projections have been hypothesized to play some role in said mechanism (see Mollick et al., 2020).

Once a cue or action has acquired some associative strength, the repeated omission of reward after its presentation allows for the restitution of behavior to the initial non-responsive state. Reward omissions following the presentation of an already conditioned stimulus (or whenever $\lambda$ is smaller than $\Sigma V_{N}$; i.e., negative reward prediction error) trigger a phasic decrease (or dip) in dopamine activity (Schultz et al., 1997; Matsumoto and Hikosaka, 2007). If repeated consistently, these dopamine dips are known to decrease dopamine firing to the target stimulus in subsequent trials. This translates into a decrease in both conditioned responses and reinforcing effects associated to the target stimulus until reaching a minimum value (see Fig. 2, right panel). Here, the procedure, process, and outcome are each referred to by the term "extinction ${ }^{1}$." Suppression of midbrain dopamine neurons induced by $\mathrm{LHb}$ activity plays a key role in this (context dependent;

\footnotetext{
${ }^{1}$ Note that the minimal response to the target stimulus observed after extinction procedures is quite labile and responding is often susceptible to bounce back after a slight contextual change (Bouton et al., 2021), this is not accounted by the Rescorla-Wagner model.
}

see Footnote 1) restauration mechanism. For example, a recent study by Donaire et al. (2019) found that LHb lesions impair extinction of an appetitive response in rats. Similarly, Zapata et al. (2017) found that pharmacological inhibition of the LHb impaired extinction of responses that were previously rewarded in the presence of a stimulus. However, this effect was selective for cocaine reward and did not impair extinction of responding previously rewarded with a sucrose solution. This finding was interpreted as an indicative of the greater difficulty in withholding responses rewarded with cocaine compared with those rewarded with sucrose. Both findings reveal the participation of the $\mathrm{LHb}$ in decreasing previously acquired behavior when a stimulus or action is no longer followed by a reward (i.e., extinction).

\section{Inducing, Testing, and Modeling Net Inhibitory Effects}

When conceptualizing conditioning trials as if a single cue is relevant for reward learning (such that $V_{X}=\Sigma V_{N}$ ) obtaining a negative value for $V_{x}$ with Equation 1 is logically impossible. As $V_{x}$ would range from zero to $\lambda$, it can only vary in its degree of "rewardingness" (for lack of a better term). That is, one could conceive of a stimulus as more or less rewarding than the other, but hardly as more aversive in a general sense. However, a distinctiveness of the Rescorla-Wagner model allows to account for both net negative associative strength values and acquisition of opposed affective effects. Such feature is important because it captures aspects of key conditioning phenomena.

A neutral stimulus that is consistently paired with the omission of an expected affectively significant event often acquires the opposite affective valence. In this case, a stimulus paired with the omission of an expected reward would acquire aversive properties (Wasserman et al., 1974). This means that the organism will be inclined to avoid it (either actively or passively) or escape from it. On the other hand, the negative summation effect occurs 


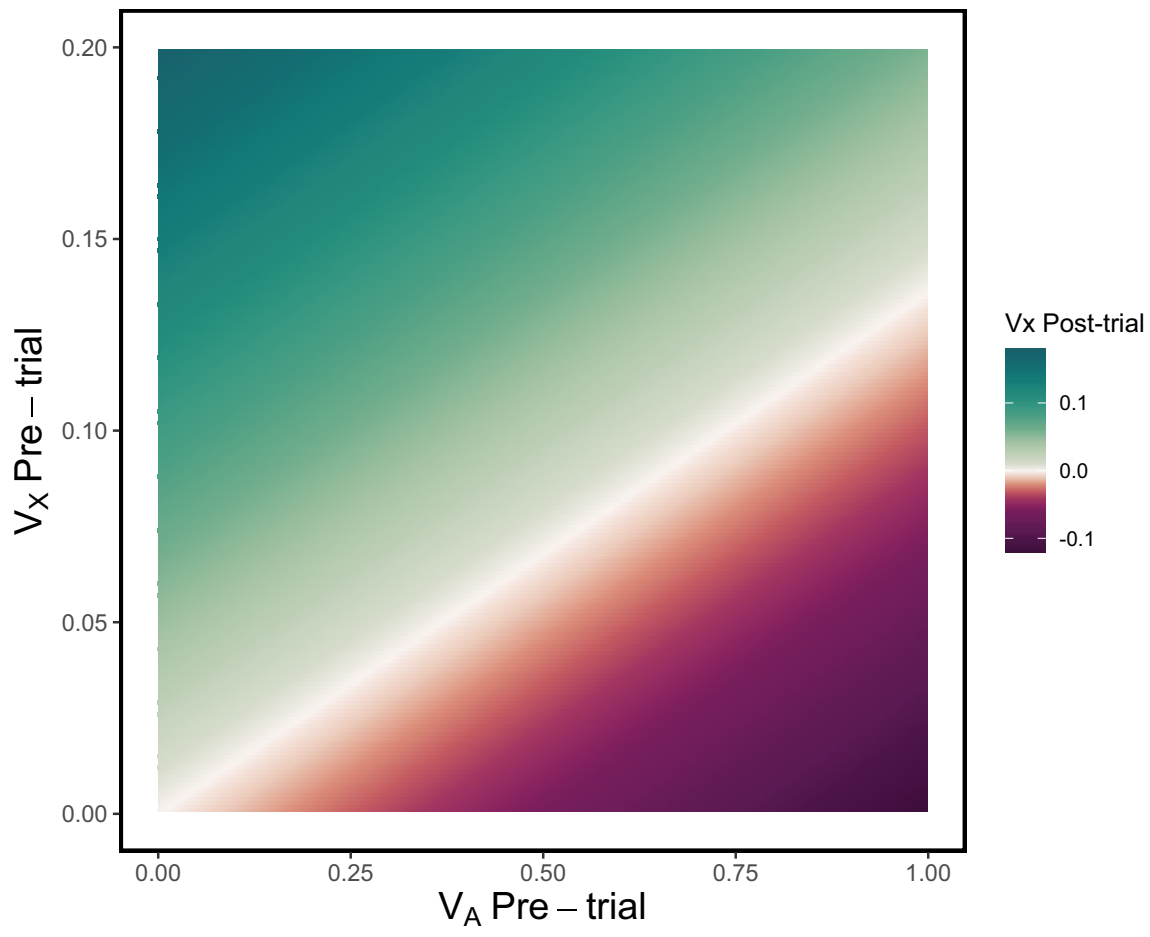

Figure 3. Possible values of associative strength for stimulus $X\left(V_{X}\right.$; color scale) after being presented in compound with an excitatory conditioned stimulus, $A$, and followed by the omission of reward $(\lambda=0)$. According to the Rescorla-Wagner model, the outcome depends on the associative strength of the companion stimulus $A$ (horizontal axis) that of $X$ (vertical axis) before the reward omission episode, and on learning rate ( $\alpha=0.12$ is assumed).

when a stimulus associated with the omission of reward is presented simultaneously with one that reliably predicts the reward. The usual result is that responding to the compound stimulus is reduced compared with that controlled by the reward-predicting stimulus alone (Acebes et al., 2012; Harris et al., 2014). The model of Rescorla and Wagner (1972) allows for partitioning of the elements that constitute a compound stimulus; concretely, it provides a rule to determine how associative strength values of different stimuli presented simultaneously interact trial by trial. Recall that $\Delta V_{X}$ represents the change in the associative strength of a single element, $X$, of the assemblage of current stimuli, $N$. Whenever $\Sigma V_{N}$ exceeds the value of $\lambda$ (as in trials involving reward omission), $V_{x}$ could take negative values under appropriate conditions. In order for $X$ to end up with a net negative associative strength following reward omission requires that (1) its current associative strength to be sufficiently low and (2) the remaining of the coextensive stimuli have some associative value (see Fig. 3).

In the Rescorla-Wagner model, the vigour of responses evoked by $\Sigma V_{N}$ is assumed to be a sort of algebraic sum of the values of current stimuli (obviating biological limits). Crucially, this model assumes that the context (cues that are continually present) could acquire associative strength and is thus capable of meaningfully impacting learning. For example, presenting $X$ without the reward and the reward without $X$ with time intervals in between would imbue that stimulus with net inhibitory properties (i.e., $V_{x}<0$ ). This protocol is known as the explicitly unpaired procedure. In such a condition, the context, $C$, acquires a positive associative strength $\left(V_{C}>0\right)$, by being present whenever the reward is delivered. Importantly, the context is also present during the trials involving the $X$ stimulus plus the omission of reward, being $\Sigma V_{N}$ equal to $V_{C}+V_{X}$ (Wagner and Rescorla, 1972). Then, $\Delta V_{X}$ will be negative for every such trial, accruing a negative value progressively over $V_{X}$. This would manifest as aversion-related behaviors toward $X$ (see Wasserman et al., 1974) and as a subtraction of the response vigour evoked by a reward-predicting stimulus whenever $X$ is presented (i.e., $\left.X \in \Sigma V_{N}<X \notin \Sigma V_{N}\right)$. Such outcomes are justified by two assumptions. First, a negative $V_{X}$ implies a subtraction of the response-evoking potential of concurrent excitatory stimuli (Wagner and Rescorla, 1972). Second, negative associative values imply that a stimulus has an affective valence that opposes that of the outcome with which it was trained (Daly and Daly, 1982).

A convenient way to conceptually and empirically instantiate these attributes of the Rescorla-Wagner model is the feature-negative discrimination protocol ${ }^{2}$. This technique consists in presenting two types of conditioning trials, usually in a random fashion. One type of trial consists

\footnotetext{
${ }^{2}$ This paradigm is more broadly known as Pavlovian conditioned inhibition; however, "conditioned inhibition" is also used to designate the process that the target stimulus undergoes (here termed "inhibitory learning"), as well as the empiric demonstration that such a process has taken place (Savastano et al. 1999); therefore, here we use the term "feature-negative discrimination" for the procedure and "inhibitory learning" for the process to avoid ambiguity.
} 


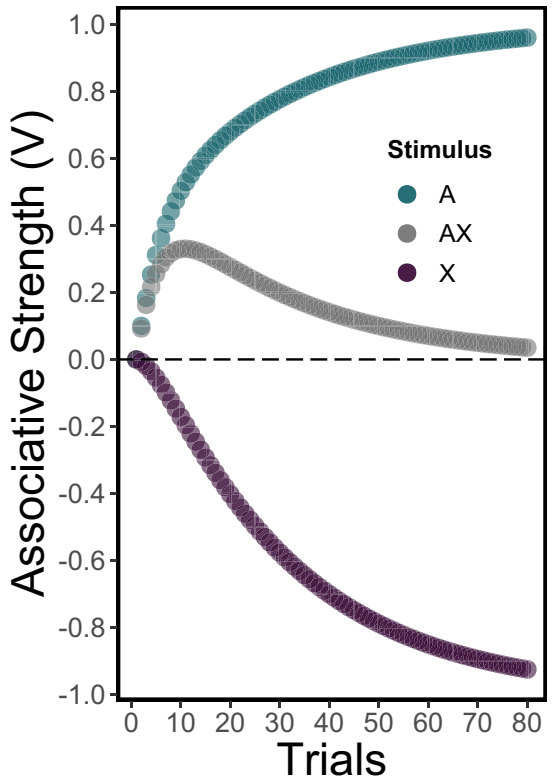

Figure 4. Associative strength, according to the RescorlaWagner model, of stimuli involved in a feature-negative discrimination protocol in 80 trials of each type. Trials of the stimulus $A$ plus reward and not rewarded $A$-with- $X$ trials were assumed to alternate non-randomly. Values of $\alpha$ were set at 0.1 and 0.075 for rewarded $(\lambda=1)$ and not rewarded $(\lambda=0)$ trials, respectively. The associative strength of the stimulus $A$ and the compound stimulus $A X$ could be observed in actual performance. In contrast, the associative strength of $X$ alone (i.e., the conditioned inhibitor) is theoretically inferred from the model and could be revealed by special tests (see text).

of presenting a single stimulus, $A$, followed by an affective outcome (e.g., reward). The remaining type of trial consists of the same stimulus accompanied by another stimulus, $X$, followed by the omission of that outcome. Such a manipulation, according to the Rescorla-Wagner model, leads stimulus $A$ to acquire a positive associative strength and stimulus $X$ to acquire a deep negative associative strength (see Fig. 4). This hypothetical attribute is known as conditioned inhibition, and Rescorla (1969) stated that it should be empirically demonstrated using two special tests. One of these tests is the above-described negative summation effect and the other is the retardation in the acquisition of a conditioned response by the target stimulus. Also, as stated above, conditioned inhibitors trained with an outcome of a particular affective valence have been documented to acquire an opposed valence (i.e., appetitive to aversive and vice versa).

\section{Dopamine Dips Propagate from Reward Omission to Events That Predict It}

The omission of an expected reward seems to promote a similar backpropagation process to the one that takes place when a cue predicts reward. Interestingly, the LHb is likely to play a crucial role in this process (see also Mollick et al., 2020). As mentioned above, reward omission triggers a phasic depression in dopamine release. In turn, this promotes a decrease in dopamine release (and its behavioral outcomes) toward cues that predict reward omissions. This implies that dopamine dips would promote a plastic change in the behaving agent which is opposite to that involved in reward. The clearest illustrations of this claim are brought by two complementary experiments to be described below.

First, Tobler et al. (2003) exposed macaque monkeys to a feature-negative discrimination protocol (see section above). In this study, the presentation of a cue alone predicted reward delivery. The presentation of the same cue accompanied by another stimulus, the conditioned inhibitor, predicted reward omission. Through training, the conditioned inhibitor acquired the capacity of counteracting the effects of the companion cue in reward omission trials (see Fig. 4, gray circles). This tendency was also observed when the conditioned inhibitor was presented along with a different reward-predicting cue in a novel compound (i. e., negative summation). Notably, those effects were reported at both behavioral (licking response) and neural (dopamine neuron activity) levels of observation. Most importantly, the presentation of the conditioned inhibitor alone, while behaviorally silent, was capable of decreasing baseline dopamine firing levels. This revealed that reward omission promotes a physiological transfer of function from the situation in which the reward is actually omitted to the cue that consistently predicts it.

The second experiment was conducted recently by Chang et al. (2018), using rats as subjects. In this study, brief dopamine dips were artificially induced in midbrain neurons following the simultaneous presentation of a reward-predicting cue and a novel cue. After training, the novel cue acquired the properties of a conditioned inhibitor, as per the conventional tests of negative summation and retardation of acquisition. Crucially, in the training trials in which this novel cue was involved the reward was not omitted. Thus, the inhibitory effects in the target cue must have arisen from the artificial dips in dopamine firing, complementing the findings of Tobler et al. (2003). On one hand, Tobler et al. (2003) demonstrated that dopamine dips could propagate from the omission of reward to the presentation of an arbitrary event; on the other hand, Chang et al. (2018) demonstrated that the dopamine dips themselves are sufficient for this to occur.

As we mentioned above, one of the most robust physiological effects of $\mathrm{LHb}$ activation is producing phasic dips in dopamine firing. Therefore, both Tobler et al. (2003)'s and Chang et al. (2018)'s findings are relevant to elucidate the function of LHb physiology in learning from experiences involving reward omission. In another recent study, Mollick et al. (2021) intended to test this notion with humans in an fMRI study. The aim of this study was to replicate Tobler et al. (2003)'s behavioral protocol but including the measurement of the activity in different regions across the whole brain (not only in midbrain dopamine cells). Mollick et al. (2021) indeed captured greater $\mathrm{LHb}$ activity during the presentation of a conditioned inhibitor compared with control stimuli; however, this difference did not survive correction for multiple comparisons. Therefore, the authors recommend further replication before any inference can be made. While an earlier 
human fMRI study (Salas et al., 2010) already documented that the habenula is activated when an expected reward is omitted (i.e., negative reward prediction error), evidence for the backpropagation of this effect remains elusive. A possible explanation for the null result reported by Mollick et al. (2021) is that the human LHb is quite small and cannot be differentiated from the medial habenula with standard fMRI resolutions (cf. Salas et al., 2010). Similarly, the study by Mollick et al. (2021) failed to capture decreased activity in midbrain dopamine regions during reward omissions. The authors argued that their fMRI spatial resolution may have also precluded the distinction between these regions and the adjacent GABAergic RTMg (which was presumably active as well during reward omissions).

\section{Concurrent Reward Cues Contribute to Dopamine Dips and Their Propagation}

The above section concluded that dopamine dips may function as "teaching" signals for gradually imbuing a stimulus with negative associative strength. The Rescorla-Wagner model states that the negative associative strength accrued by a stimulus that is paired with reward omission depends on concurrent reward cues (see Fig. 3). Therefore, if we assume that dopamine dips depend on LHb activation, the latter would rely on current information about an impending reward. That is, if a stimulus is followed by the omission of reward, its associative strength would not change unless it is coextensive with reward-related cues. Therefore, LHb activation might not be induced by the sheer non-occurrence of reward. Rather, the LHb may become active when this non-occurrence coincides with signals from other brain regions indicating probable reward. Furthermore, the greater the signal for reward the larger the downshift in associative strength of (and the aversion imbued to) the stimulus paired with reward omission (see Fig. 3). Accordingly, dopamine dips induced by reward omission would be proportional to the probability (or magnitude) of the reward associated with a conditioned stimulus. Tian and Uchida (2015) found evidence supporting this assumption using mice as subjects and three different probabilities of reward associated with different target stimuli. Crucially, this pattern of results was hindered in animals with bilateral lesions in the whole habenular complex.

A relevant consideration is the origin of the signals required for invigorating the LHb activity on signals of an impending reward. The entopeduncular nucleus (EPN; border region of the globus pallidus internal segment in primates; Hong and Hikosaka, 2008) and lateral hypothalamus (LH; Stamatakis et al., 2016) provide excitatory inputs to the LHb. However, up to this point, it remains unclear how impending reward signals mediate the invigoration of the LHb. An elegant study with macaques by Hong and Hikosaka (2013) found that electric stimulation of the ventral pallidum (VP) consistently inhibited the activity of the LHb. These authors conjectured that an interaction between inputs from the EPN and the VP in the LHb was responsible for the activation of the LHb. Recent evidence indicates that GABAergic neurons in the VP are activated by surprising rewards and reward-predicting cues and inhibited by aversive stimuli and reward omission (Wheeler and Carelli, 2006; Stephenson-Jones et al., 2020). Crucially, inhibition of LHb projecting VP GABAergic neurons on reward omission depends on current reward cues. In short, these VP neurons showed a biphasic ascending $\rightarrow$ descending pattern of activation to the rewardcue $\rightarrow$ reward-omission sequence. Therefore, this structure is a strong candidate for providing signals to invigorate the activation of the $\mathrm{LHb}$ in reward omission episodes via disinhibition.

The strength with which the EPN triggers LHb activation may depend on the prior tone of VP inhibitory inputs. In fact, Stephenson-Jones et al. (2020) reported that a number of GABAergic VP neurons synapsing with the $\mathrm{LHb}$ exhibited a sustained pattern of activation during rewardpredicting cues. Then, sudden inhibition of these neurons upon reward omission may combine with activation of EPN signals for strongly disinhibiting the LHb. Stephenson-Jones et al. (2020) also observed that a small subpopulation of LHb-projecting glutamatergic VP neurons showed the opposite pattern of activation to that of GABAergic ones. Activation of these neurons may add up to complement the potent disinhibition-excitation process in the $\mathrm{LHb}$ during omission of an expected reward.

Knowing the source of excitatory and inhibitory inputs to the LHb is an essential matter. However, this leads to the task of identifying the upstream inputs of these sources (Bromberg-Martin et al., 2010) and so on. Hong and Hikosaka (2013) speculated that the EPN may activate the LHb through disinhibitory inputs from striosomal regions of the dorsal striatum. This conjecture has recently been supported by a study conducted by Hong et al. (2019), in which the activation of striatal neurons inside or nearby striosomes correlated with activation of the LHb. Hong et al. (2019) further advanced the hypothesis that striosomes may convey both excitatory and inhibitory inputs to the LHb. Regarding the VP, Stephenson-Jones et al. (2020) reported that neurons in this structure targeting the $\mathrm{LHb}$ became active in a state-dependent fashion; specifically, these neurons fire according to the motivational status of the subjects, which is probably mediated by hypothalamic signals. It seems reasonable that activation of the $\mathrm{LHb}$ would be dependent on the energetic supply status or sexual drive at a particular moment. In short, the omission of reward should not cause substantial disturbance to a sated individual. This could be another possibility for explaining the failure of Mollick et al. (2021) to replicate Tobler et al. (2003)'s findings. Both studies used fruit juice as reward. However, in the latter study macaques were liquid deprived, while in the former participants were recruited based on self-reported preference for the type of juice employed. The motivational state of participants in Mollick et al. (2021)'s study was probably insufficient to induce strong physiological responses in the target regions. 


\section{The LHb Promotes Stimulus-Specific and Response-Specific Inhibitory Effects}

Although feature-negative discrimination is a prototypic procedure for inducing conditioned inhibition effects in appetitive settings (which here we are proposing as a behavioral phenotypic model of LHb function), other protocols are also capable of doing so (see Savastano et al., 1999). Likewise, other behavioral manifestations, besides negative summation and retardation of acquisition tests, could be used to certify conditioned inhibition (Wasserman et al., 1974). For example, in a pivotal study, Laurent et al. (2017) exposed rats to a backward conditioning preparation to induce conditioned inhibition. Then, conditioned inhibitory properties of stimuli were tested through a Pavlovian-to-instrumental transfer (PIT) test. This backward conditioning procedure consisted in presenting one of two different auditory target stimuli following, rather than preceding, as typically done, the presentation of two different rewards; each target stimuli was consistently associated with its corresponding reward in a backward fashion (reward $\rightarrow$ stimulus). Next, rats pressed two levers to obtain rewards and each of the rewards used in the previous phase was associated with a different lever. In the PIT test, the levers were present but pressing was no longer followed by reward. In that condition, the target stimuli that were used in the first phase were presented randomly and lever pressing was recorded. Conditioned inhibition was revealed inasmuch each target stimulus biased lever-pressing toward the opposite lever than that associated with its corresponding reward. Importantly, Laurent et al. (2017) found that this outcome was impaired by bilateral lesions of the $\mathrm{LHb}$.

The disruption of conditioned inhibition was first observed by performing an electrolytic lesion in the entire $\mathrm{LHb}$. This result was replicated when the authors induced a selective ablation of neurons in the $\mathrm{LHb}$ that projected to the RMTg via a viral technique. Therefore, the authors concluded that this LHb-RMTg pathway is crucial for learning these specific negative predictions about rewards. Remarkably, electrolytic lesions in the LHb did not disrupt PIT performance for rats trained with traditional forward-conditioning (stimulus $\rightarrow$ reward) procedure. This suggests that the disruptive effect was confined to inhibitory learning (see also Zapata et al., 2017). Laurent et al. (2017)'s findings provide important insights for understanding the role of LHb-RMTg pathway in the development of conditioned inhibition in appetitive settings. Beyond generally impairing responding when reward is negatively predicted, the LHb seems to promote learning to perform specific action patterns to specific cues. Apparently, this structure participates in enabling alternative behaviors for exploiting alternative resources in the environment when the omission of a particular reward is imminent.

Notably, the findings reported by Laurent et al. (2017) challenge the Rescorla-Wagner model. According to this model, a stimulus that is explicitly unpaired with a reward can acquire a negative associative strength (i.e., become a conditioned inhibitor). This would be mediated by the relatively high associative strength in the context that coextends with the target stimulus preceding reward omission. The
Rescorla-Wagner model predicts that backward conditioning would lead as well to conditioned inhibition by this rationale (see Wagner and Rescorla, 1972). In backward conditioning, the target stimulus also co-occurs with the presumably excitatory context. However, Laurent et al. (2017) observed specific inhibitory effects for each of the target stimuli in their study. It is worth stressing that those target stimuli were trained at the same time range and in the same context. Thus, the target stimuli presumably acquired conditioned inhibitory properties by being (negatively) associated with each reward. In contrast, the Rescorla-Wagner model predicts that both target stimuli would acquire general inhibitory properties. This follows from the rationale that each target stimulus was paired with the absence of either reward in a context associated with both.

At the behavioral level, Laurent et al. (2017)'s findings can be readily accounted for by the sometimes-opponent-process (SOP) model (see Vogel et al., 2019). This associative learning model invokes stimuli after-effects that are capable of shaping behavior if they are appropriately paired with other events. According to the SOP model, there is an inhibitory (opponent) decaying trace of its own kind following the delivery of each particular reward. In the study of Laurent et al. (2017), each reward's unique trace would have been paired with each of the target stimuli. As a consequence, each target stimulus acquired reward-specific conditioned inhibitory properties. As LHb lesions completely abolished the behavioral effect resulting from this protocol, this structure appears to play a crucial role in this phenomenon. However, the putative physiological means by which LHb mediates learning in conditions such as those in Laurent et al. (2017)'s study remain to be determined.

\section{The LHb Participates in Inhibitory Learning Even without Temporally Specific Reward Omission}

The findings reported by Laurent et al. (2017) merit further examination. Based on this study, we can assume that the LHb could exert its effects without acute episodes involving the omission of an expected reward. Such a process is also instantiated in explicitly unpaired procedures, in which a target stimulus acquires inhibitory properties solely by alternating with reward. A recent study by Choi et al. (2020) sought to test the hypothesis that the $\mathrm{LHb}$ is involved in learning the association of a cue with the absence of reward. These authors exposed rats to an explicitly unpaired conditioning procedure that used a light as a negative predictor of food delivery. Choi et al. (2020) found increased c-Fos expression in the LHb of rats exposed to an explicitly unpaired conditioning procedure compared to controls. This result supports the idea that the $\mathrm{LHb}$ is engaged in the learning process that takes place when a stimulus signals the absence of reward. In a separate experiment, these authors did not find any disruption in performance on this protocol when they induced excitotoxic LHb lesions. However, this should not be taken as negative evidence of the involvement of the $\mathrm{LHb}$ in inhibitory learning. This finding may be explained on the basis of the need for special tests (e.g., 
summation, retardation, PIT; see Rescorla, 1969) to certify that an inhibitory stimulus is capable to counteract reward-related behaviors. Unfortunately, Choi et al. (2020) did not conduct any of the available tests for assessing conditioned inhibition.

Both in backward conditioning and in explicitly unpaired conditioning preparations, the reward would not be expected to occur in any particular moment; specifically, a reward delivery is scheduled to occur at random intervals without discrete cues anticipating it, unlike in extinction and in feature-negative discrimination procedures. The ability of the LHb to modulate reward seeking, even in paradigms not involving explicit reward omissions, raise the question of what the mechanisms involved are. A recent study by Wang et al. (2017) may shed light in this issue. These authors described a rebound excitation in the LHb following the inhibition typically produced by reward delivery. Given appropriate timing, the traces of such a rebound excitation could have been paired with the target stimuli for the control, but not for the lesioned, subjects in Laurent et al. (2017)'s study. Such pairings would facilitate the propagation of $\mathrm{LHb}$ excitation, presumably promoting dopamine suppression, eventually on the target stimuli alone. However, this mechanism cannot account readily for inhibitory learning in explicitly unpaired conditioning procedures. In these protocols, the target stimulus does not consistently follow the reward, but these events are rather separated by intertrial intervals of varying durations. Therefore, decreases in dopamine mediated by rebound activity of the LHb could hardly explain the inhibitory properties acquired by a target stimulus in explicitly unpaired procedures.

The $\mathrm{LHb}$ is known to modulate other monoamines besides dopamine, such as serotonin (Amat et al., 2001). Tonic serotoninergic activity depends on accumulated experience with rewards (Cohen et al., 2015). In turn, serotonergic tone determines the effects of phasic activity in serotonergic neurons, most of which is known to occur during aversive experiences (Cools et al., 2011). Thus, the $\mathrm{LHb}$ may participate in accumulating information about reward probability in a given environment. This would tune serotonin levels during inter-reward intervals on explicitly unpaired procedures, which might determine the affectiveness of salient cues throughout those intervals. A similar effect may also be prompted by the LHb via modulation of tonic dopamine levels (see Lecourtier et al., 2008), which would not contradict with the serotonergic hypothesis. However, it is yet to be determined with certainty whether the LHb participates in inhibitory learning from explicitly unpaired protocols. This will require using either in vivo real-time recording or special tests for conditioned inhibition in lesion/inactivation studies. In addition, it is not clear how the LHb would be more active in explicitly unpaired conditions than in control conditions involving equivalent reward density, which was the main result reported by Choi et al. (2020). A possibility is that the LHb is relatively inactive when a reward is fully predicted by a cue. Conversely, it may be tonically active in ambiguous conditions; for example, when the reward or the cue occur at any given time without notice (i.e., are explicitly unpaired).

\section{The Habenulo-Meso-Cortical Excitatory Pathway May Play a Role in Negative Reward Prediction Error}

The glutamatergic LHb-VTA-mPFC pathway (see Fig. 1) is less well-known than the LHb-RMTg pathway but may also play an important role in inhibitory learning from reward omission. As we described earlier, unlike other $\mathrm{LHb}$ efferents this pathway promotes, rather than inhibiting, dopaminergic transmission. Perhaps, the most crucial evidence supporting this idea stems from a study conducted by Lammel et al. (2012). These authors reported that blocking dopaminergic transmission in the mPFC abolishes the capacity of the $\mathrm{LHb}$ to generate aversion to spatial stimuli. Therefore, this pathway may be crucial in complementing dips in meso-striatal dopaminergic release for learning from negative reward prediction errors. Two putative, not mutually exclusive, mechanisms for such an effect could be (1) directly opposing midbrain dopamine activity (see Jo et al., 2013) and (2) selecting which stimuli should be filtered to control behavior (see Vander Weele et al., 2018). The connectivity relationship between the MPFC and the LHb is reciprocal (see Mathis et al., 2017) and their afferences also converge in certain brain locations (Varga et al., 2003). The mPFC has long been considered a key brain region for restraining inappropriate actions and adjusting behavior when errors occur (Ragozzino et al., 1999) but only recently this has been considered from an associative learning perspective. Although research on LHb-mPFC interaction is still scant, we outline some ideas on how these regions may jointly contribute to learning from negative reward prediction errors.

We should consider first that the MPFC is divided in functionally dissociable anatomic subregions, at least for some eutherian mammals (see Ongur and Price, 2000). A subregion that might play a role in the LHb's network is the prelimbic cortex of rodents, which presumably corresponds to the pregenual anterior cingulate in primates (see Laubach et al., 2018). Several studies have linked this subregion with the ability of mammals to restrain a dominant (previously rewarded) response (Laubach et al., 2015). An outstanding example of this is a study conducted by Meyer and Bucci (2014) using rats as subjects. These authors found that prelimbic, but not in the adjacent infralimbic, mPFC lesions impaired inhibitory learning process in an appetitive feature-negative discrimination paradigm in rats (i.e., differentiation in responding to $A$ and AX in Fig. 4). As we described above, this paradigm consists in presenting a target stimulus that signals reward omission. However, importantly, this target stimulus occurs in the presence of a cue that otherwise consistently predicts reward delivery. To effectively learn how to stop reward-related responses in such situation, subjects must solve the conflict between reward and reward-omission cues that are presented simultaneously.

It has been suggested that the opposing effects of the prelimbic division of the MPFC upon the reward systems is exerted via an aversion mechanism. This rationale is supported by evidence that this region is involved in fear learning (Burgos-Robles et al., 2009; Piantadosi et al., 
2020) and exhibits robust excitatory connections with the basal amygdala (Sotres-Bayon et al., 2012). Conversely, the infralimbic portion of the mPFC, has been related to both the expression of habitual reward-related responses (Haddon and Killcross, 2011) and fear suppression (Santini et al., 2008; but see Capuzzo and Floresco, 2020). The involvement of the infralimbic cortex in the latter of these processes could be accounted for in terms of mediation of a subjective relief state. Such a state would be functionally equivalent to reward and, therefore, opposed to aversive states in a hierarchical fashion under appropriate circumstances.

However, some findings (e.g., Sharpe and Killcross, 2015) seem to contradict this prelimbic-aversive and infralimbic-appetitive notion, which prompts deviations from this theoretical scheme. Instead of the aversive-appetitive dichotomy, Sharpe and Killcross (2018) sustain that the infralimbic cortex promotes attention to stimuli that reliably predict affectively significant events, regardless of their valence (i.e., reflective control). In contrast, the prelimbic cortex would promote attention to higher order cues setting hierarchical (as in conditional probabilities) relationships between stimuli and relevant outcomes (i.e., proactive control), again, regardless of their affective valence. A similar point has been raised by Hardung et al. (2017), who found evidence for a functional gradient in the mPFC spanning from the prelimbic to the lateral-orbital cortex (passing through the infralimbic, medial-orbital and ventral-orbital regions). These authors reported that regions near to the prelimbic cortex tend to participate more in proactive control, while regions near the lateralorbital cortex participate more in reflective control. However, this study was conducted in an appetitive setting, so hierarchical control (reactive vs proactive) and opponent affective control (appetitive vs aversive) hypotheses cannot be disambiguated. While cortical inputs to the LHb are generally modest, the prelimbic cortex makes the largest contribution among the regions of the cortex innervating this structure (Yetnikoff et al., 2015). This may indicate that this pathway serves as an either positive or negative feedback mechanism, depending on whether these connections are excitatory or inhibitory. Being the LHb a major aversive center, the former possibility would be at odds with the approach of Sharpe and Killcross (2018).

Furthermore, a recent finding has tilted the scale in favor of the aversive-appetitive modularity of the mPFC. Yan et al. (2019) reported that spiking in a subpopulation of neurons in rats dorsomedial PFC (dmPFC; including prelimbic and dorsal cingulate cortex) was (1) increased upon the presentation of a cue signaling an imminent electric shock, and (2) increased, but considerably less, and then decayed below baseline on the presentation of a cue that signals the omission of the shock. In addition, Yan et al. (2019) showed that the cue signaling shock omission passed the tests of summation and retardation for conditioned inhibition, both at neural and behavioral levels. Interestingly, this pattern of results mirrors those in the study by Tobler et al. (2003) in an appetitive setting with macaques. In brief, suppressing fear responses involves inhibition of dmPFC neurons, suggesting that this region serves primarily to facilitate aversive learning rather than having a general hierarchical control function. This is in line with the idea that LHb input to the prelimbic cortex may counteract the effects of reward cues via an opponent affective process. Such a mechanism has been regarded as one of the necessary conditions for the occurrence of inhibitory learning and performance (see Konorski, 1948; Pearce and Hall, 1980). However, this mechanism may be complemented with others, such as threshold increase and attention to informative cues (Laubach et al., 2015).

\section{Relevance of the LHb for Mental Health}

There is an emerging literature suggesting that many psychiatric disorders can be characterized as alterations of the ability to predict rewards and aversive outcomes (Byrom and Murphy, 2018). Therefore, human wellbeing could be understood, at least in part, in terms of the dynamics of associative learning and outcome prediction (Papalini et al., 2020). Thus, disrupting the neural underpinnings of outcome predictions, either in an upward or downward direction, could lead to maladaptive behaviors that threaten people's subjective wellbeing. Disappointment, frustration, and discomfort stemming from worse than expected outcomes are useful for directing behavior to adaptive ways of interaction with our surroundings (see Fig. 5, light purple arrows). However, if these subjective sensations are lacking or excessive, it is likely that problematic behaviors will arise (see Fig. 5, dark colored arrows).

Some authors have proposed that a pervasive reward learning process (Chow et al., 2017; Sosa and dos Santos, 2019a) can explain maladaptive decision-making in impulsive and risk-taking behaviors. This may arise in part from a deficit in learning from negative reward prediction errors (Laude et al., 2014; Sosa and dos Santos, 2019b; see Fig. 5, dark aqua arrow). Impulsive-like behavior is often studied in choice protocols with animal models. A study by Stopper and Floresco (2014) showed that $\mathrm{LHb}$ inactivation led to indifference when rats choose between ambiguous alternatives. Specifically, subjects were biased away from the most profitable of two alternative rewards when a delay was imposed between the selecting action and that reward; however, this bias was abolished by $\mathrm{LHb}$ inactivation. This suggests that delay aversion might stem from a negative reward prediction error mechanism, rendering the puzzle even more complex. On the other hand, studies involving humans and rodent models of helplessness (Gold and Kadriu, 2019; Jakobs et al., 2019) have suggested that an over-reactivity of the LHb can induce symptoms associated with depression (see Fig. 5, dark purple arrows). Such symptoms include reluctance to explore new environments and unwillingness to initiate challenging actions, which could be interpreted as a constant state of disappointment or sense of doom (Proulx et al., 2014). In that case, atypically high reward expectations could lead to a pervasive and maladaptive enhanced inhibitory learning. Another possibility is that some individuals may have a proclivity to 


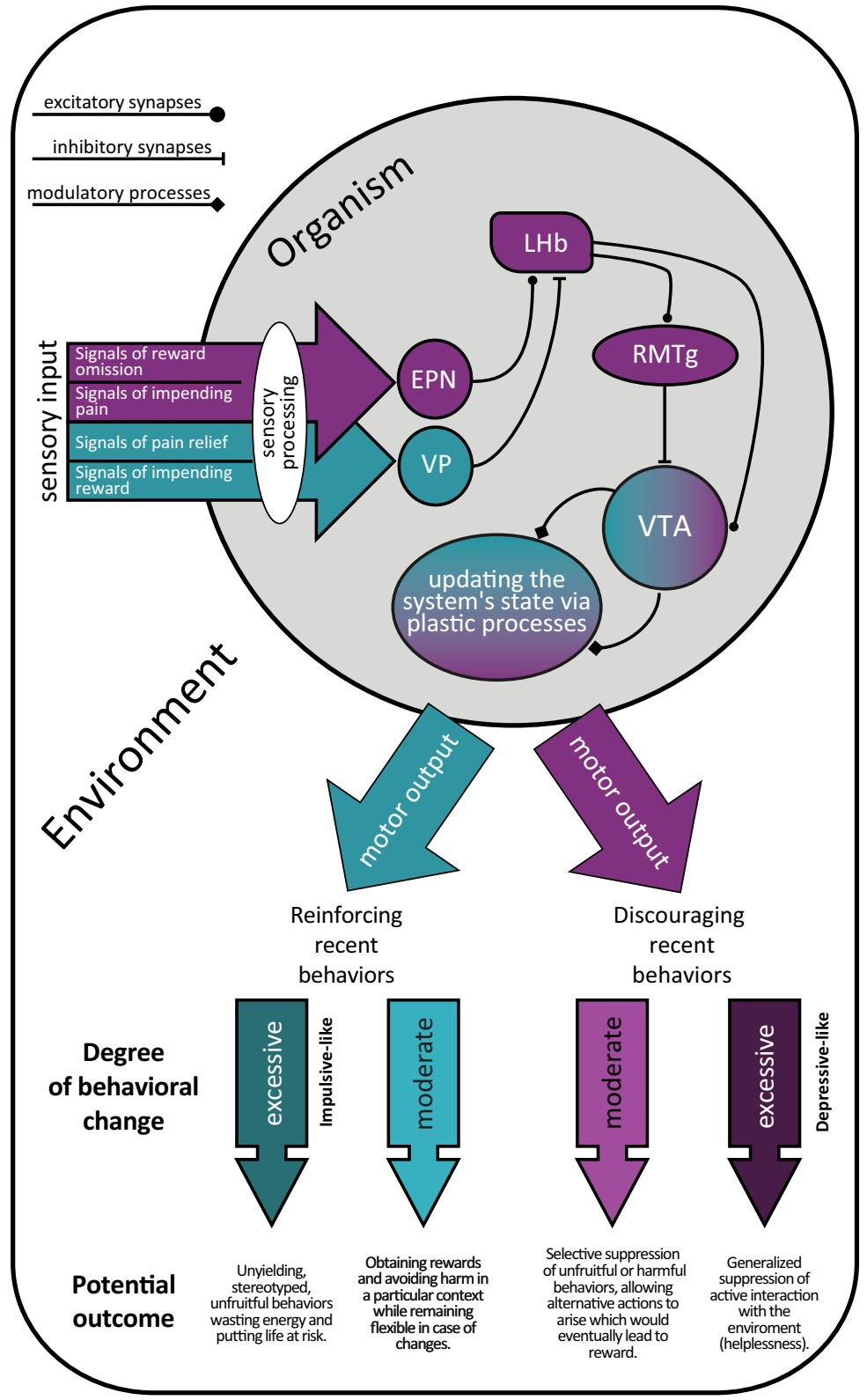

Figure 5. Simplified diagram representing the participation of the LHb in plastic brain processes for adaptively updating behavior and hypothesized consequences of excessive activity (dark purple) or inactivity (dark aqua) of this brain structure.

overgeneralize learning from negative reward prediction errors, which would cause a decreased activity overall (a major symptom of depression). These putative mechanisms are, of course, not mutually exclusive.

Synthetizing behavioral and neurophysiological studies could serve to address the etiology and phenomenology of these psychiatric conditions. Such an enterprise would require integrating details regarding behavioral changes in response to environmental constraints and the underlying neurobiological mechanisms involved. This is precisely what we have attempted in the present article. If ongoing research continues to make advances, then this perspective could serve to guide clinical practice. An example would be determining whether psychotherapy would be enough to tackle a particular case or whether combining it with pharmacotherapy would be pertinent. Knowledge about neural and behavioral dynamics might serve to design combined intensive interventions to avoid long-term side effects of medications and dependency (Hengartner et al., 2019). Additionally, understanding morphogenesis and wiring of the LHb during embryological development could be relevant to inquire whether disturbances in these processes underlie problematic behavioral traits (Schmidt and Pasterkamp, 2017).

Some recent studies have furthered our understanding of behavioral-physiological interactions involving the $\mathrm{LH}$ from basic science to application. For example, Zhu et al. (2019) found an association between subclinical depression and atypical connections in the dorsal posterior 
thalamus and the LHb. This peculiar connectivity of the $\mathrm{LHb}$ might predispose people to depression or, alternatively, some life events could promote behavioral patterns leading to that phenotype. Experiences of reward loss could vary regarding the time frame and magnitude of the involved rewards (Papini et al., 2006). A person could lose money during a gambling night with friends, which could be a relatively innocuous experience, or lose a lifetime partner, being a devastating event. Significant life experiences can cause detectable changes in brain networks (Matthews et al., 2016) and tracking these changes can lead to finding ways to understand the underpinnings of behavioral phenotypes associated with psychiatric conditions. An intriguing example is that attenuating $\mathrm{LHb}$ activity has been shown to ameliorate depressive-like symptoms induced by maternal separation in mice (Tchenio et al., 2017).

\section{Directions for Moving the Field Forward}

Computational modeling of associative phenomena is just one possible approach to account for the relationship between LHb physiology and inhibitory learning. There are other alternatives for improving our understanding of this topic, even in a more detailed manner, such as neural network models. These are powerful tools for envisaging plausible mechanisms implemented by biological agents to interact adaptively with their environment. A clear advantage of these models is that they make physiological hypotheses more tenable (Frank, 2005). To our knowledge, only few recently proposed neural-network models (see Vitay and Hamker, 2014; Mollick et al., 2020) have incorporated nodes regarding the physiology of the LHb. Even so, those models do not account for the multifaceted circuitry (e.g., including the LHb-VTA-mPFC pathway) of this structure which, we argue, is highly relevant for an utter understanding of its role in inhibitory learning. Lack of inclusion of the LHb into neural network modeling (Collins and Frank, 2014; Burgos and García-Leal, 2015; Sutton and Barto, 2018; O'Reilly et al., 2019) may be partly because of an overemphasis in performance over learning of inhibitory control. Cortical-thalamic-striatal circuits are often invoked to account for inhibitory performance, often without formally specifying how outcomes shape future actions (Verbruggen et al., 2014; van Wijk et al., 2020). An integration of learning and performance models should be pursued to increase our understanding of the broad network in which the LHb participates.

Another matter for future consideration is whether the role of the LHb in learning from reward omission is exclusive for edible rewards or it extends to other types of reward, such as sexual stimuli for receptive individuals. In addition, our understanding of the behavioral neuroscience regarding the $\mathrm{LHb}$ come from select model species. It has been hypothesized that the habenular complex evolved in an ancestor of vertebrates to enable circadian-determined movement modulation (Hikosaka, 2010). In this sense, the LHb may have later evolved its role for suppressing specific actions in response to more dynamic environmental information. Such exaptation hypothesis implies that the habenula of basal lineages did not play the same role it does in extant vertebrates. That, in turn, implies that either basal vertebrate lineages could not learn from reward omission or that they did so by recruiting other mechanisms.

Even invertebrates possess mechanisms for learning from situations involving negative prediction errors (i.e., conditioned inhibition; Britton and Farley, 1999; Couvillon et al., 2003; Acebes et al., 2012; Durrieu et al., 2020). Although this fact is appealing, for now it is fairly limited to the behavioral level of observation. However, an emerging field of research is currently scrutinizing the neural underpinnings of negative reward prediction errors in Drosophila flies (Felsenberg et al., 2017). In these animals, a bilateral structure known as the mushroom body supports associative learning using dopamine as a plasticity factor, in a similar fashion as the vertebrate brain does (Aso et al., 2014). The mushroom body possesses different subdivisions, which selectively release dopamine during reward or aversive stimuli. Intriguingly, Felsenberg et al. (2017) reported that inactivating aversion-related dopamine neurons preclude the bias in behavior otherwise produced by pairing a stimulus with reward omission. It has been suggested that aversive dopamine neurons directly oppose to reward-related dopamine neurons in the mushroom body (Perisse et al., 2016). This may indicate that invertebrate nervous systems possess the hierarchical architecture necessary for inhibitory learning, such as that found in vertebrates. Remarkably, these diverging nervous systems also exhibit antagonistic dopaminergic subsystems, similar to those that the LHb orchestrate in the vertebrate brain. Future research in this field may uncover the evolutionary origins of negative reward prediction errors and a more precise dating of the origins and precursors of the $\mathrm{LHb}$.

\section{Summary and Concluding Remarks}

Organisms benefit from possessing mechanisms to track resources in their surroundings and adjust their actions to obtain maximum profit. This relies on a delicate balance between responding and withholding specific responses whenever it is appropriate. Sensorimotor feedforward loops are mediated by long-term potentiation in some locations of the striatum induced by midbrain dopaminergic inputs (Yagishita et al., 2014). Conversely, there are several processes that prevent spurious sensorimotor loops, which would potentially waste energy and put the organism at risk. A convenient paradigm to study one such process is conditioned inhibition, a subclass of Pavlovian learning phenomena. This paradigm could serve as a principled and physiologically informed behavioral phenotype model of negative error prediction. Negative feedback control mechanisms had been recently proposed to be of primary importance to understand adaptive behavior (Yin, 2020). Therefore, conditioned inhibition might be a particularly relevant and timely conceptual and methodological tool. Intriguingly, although inhibitory learning has been thought to be multifaceted in nature (Sosa and Ramírez, 2019), several of its manifestations seem to implicate the LHb. 
Omission of expected rewards at a precise time promotes dopamine dips in canonical midbrain neurons with a remarkable contribution of the LHb (see Mollick et al., 2020). These dopamine dips back-propagate to stimuli that consistently predict reward omission in a way that resembles plastic changes associated with dopamine release (Tobler et al., 2003). All things being equal, dopamine dips can counteract phasic dopamine effects on behavior (Chang et al., 2018). This accounts for LHb's contribution to extinction of a previously acquired response (Zapata et al., 2017), feature negative discrimination, negative summation, and retardation of acquisition in appetitive settings (Tobler et al., 2003). However, although the activity of the LHb is associated with dopamine dips, it may also participate in learning processes beyond general suppression of previously rewarded actions (Laurent et al., 2017). This selective effect is remarkable given that other brain areas have been associated with global response inhibition (see Wiecki and Frank, 2013).

Even if LHb's suppressing effects on midbrain dopamine neurons are robust, the excitatory LHb-VTA-mPFC pathway may also play a role in inhibitory learning from reward omission. Lesions of the prelimbic portion of the mPFC impair inhibitory learning in appetitive settings (Meyer and Bucci, 2014). This region receives indirect dopaminergic input from the $\mathrm{LHb}$ and its activation has been implicated in aversion learning (Lammel et al., 2012). Some learning theories have proposed that conditioned inhibition is partly determined by antagonizing the effect of an affectively loaded conditioned stimulus (Konorski, 1948; Pearce and Hall, 1980). The activity of this pathway leads to dopamine release in the MPFC, which may allow channeling relevant sensory inputs to key plastic brain regions (Vander Weele et al., 2018). Therefore, interactions of the LHb with aversive centers via the prelimbic cortex may facilitate imbuing cues with the capacity of counteracting reward-seeking tendencies. Disrupting either the excitatory or inhibitory LHb pathways has been shown to hamper inhibitory learning processes to some degree. Whether and how those pathways may interact or complement each other to promote inhibitory learning remains a matter of further investigation.

Feature-negative discrimination and extinction procedures promote a decrease (or even net negative values) in associative strength, presumably through the omission of a reward at a precise moment following a cue. However, backward conditioning also induces conditioned inhibition and this effect is impaired by specific ablation of the LHb-RMTg pathway (Laurent et al., 2017). In this paradigm, the reward is not expected at a specific point in time, so other mechanisms may be recruited by the LHb. A potential candidate for this process is the rebound activity of the LHb following inhibition by reward (Wang et al., 2017). However, alternating a target stimulus with reward delivery in an explicitly unpaired fashion also induces conditioned inhibition. There is ex-vivo evidence that such conditions promote meaningful activity in LHb neurons (Choi et al., 2020). In such case, learning could not be clearly linked neither to phasic decreases in dopamine in response to unexpected reward omission nor to postreward rebound activity of the $\mathrm{LHb}$; therefore, other mechanisms involving the LHb may take place.

Converging lines of evidence suggest that $\mathrm{LHb}$ participates in the induction and expression of inhibitory learning under different conditions involving reward. This supports the intriguing idea that this structure is composed of several parallel circuits operating in different functionally equivalent situations (Stephenson-Jones et al., 2020). Unfortunately, conditioned inhibition, the accumulated outcome of inhibitory learning, is an elusive phenomenon which often requires special tests to be validated. Moreover, some authors have claimed that each test requires up to several control conditions to be deemed conclusive (Papini and Bitterman, 1993). This complicates the matter further, as it multiplies the number of subjects that are needed to evaluate the role of the $\mathrm{LHb}$ in this phenomenon; aside from comparing the role of its different subcircuits. However, this topic is still worth investigating, as it seems to participate in many situations involving negative predictive relationships between events. Perhaps, one situation in which such processes take place is the adaptation to environments that require behavior flexibility. If one takes "negative reward prediction errors" in a broader sense, many tasks requiring shifts in behavior following subtle cues could be considered in this category. Accordingly, there is an increasing amount of evidence that LHb plays a role in updating behavior on those tasks (Baker et al., 2017). Conditioned inhibition could be conceived as an accumulated outcome of the mechanisms involved in shifting behavior in dynamic conditions by error corrections. Models of associative learning would be useful to frame and test this and further hypothetical propositions.

\section{References}

Acebes F, Solar P, Moris J, Loy I (2012) Associative learning phenomena in the snail (Helix aspersa): conditioned inhibition. Learn Behav 40:34-41.

Amat J, Sparks PD, Matus-Amat P, Griggs J, Watkins LR, Maier SF (2001) The role of the habenular complex in the elevation of dorsal raphe nucleus serotonin and the changes in the behavioral responses produced by uncontrollable stress. Brain Res 917:118126.

Aso Y, Hattori D, Yu Y, Johnston RM, lyer NA, Ngo T-TB, Dionne H, Abbott LF, Axel R, Tanimoto H, Rubin GM (2014) The neuronal architecture of the mushroom body provides a logic for associative learning. Elife 3:e04577.

Baker PM, Jhou T, Li B, Matsumoto M, Mizumori SJY, StephensonJones M, Vicentic A (2016) The lateral habenula circuitry: reward processing and cognitive control. J Neurosci 36:11482-11488.

Baker PM, Raynor SA, Francis NT, Mizumori SJY (2017) Lateral habenula integration of proactive and retroactive information mediates behavioral flexibility. Neuroscience 345:89-98.

Barrot M, Sesack SR, Georges F, Pistis M, Hong S, Jhou TC (2012) Braking dopamine systems: a new GABA master structure for mesolimbic and nigrostriatal functions. J Neurosci 32:1409414101

Bouton ME, Thrailkill EA, Trask S, Alfaro S (2020) Correction of response error versus stimulus error in the extinction of discriminated operant learning. J Exp Psychol Anim Learn Cog 46:398407. 
Bouton ME, Maren S, McNally GP (2021) Behavioral and neurobiological mechanisms of Pavlovian and instrumental extinction learning. Physiol Rev 101:611-681.

Britton G, Farley J (1999) Behavioral and neural bases of noncoincidence learning in Hermissenda. J Neurosci 19:9126-9132.

Bromberg-Martin ES, Matsumoto M, Hong S, Hikosaka O (2010) A pallidus-habenula-dopamine pathway signals inferred stimulus values. J Neurophysiol 104:1068-1076.

Burgos JE, García-Leal Ó (2015) Autoshaped choice in artificial neural networks: implications for behavioral economics and neuroeconomics. Behav Processes 114:63-71.

Burgos-Robles A, Vidal-Gonzalez I, Quirk GJ (2009) Sustained conditioned responses in prelimbic prefrontal neurons are correlated with fear expression and extinction failure. J Neurosci 29:84748482.

Byrom NC, Murphy RA (2018) Individual differences are more than a gene $\times$ environment interaction: the role of learning. J Exp Psychol Anim Learn Cogn 44:36-55.

Capuzzo G, Floresco SB (2020) Prelimbic and infralimbic prefrontal regulation of active and inhibitory avoidance and reward-seeking. J Neurosci 40:4773-4787.

Chang CY, Gardner MPH, Conroy JC, Whitaker LR, Schoenbaum G (2018) Brief, but not prolonged, pauses in the firing of midbrain dopamine neurons are sufficient to produce a conditioned inhibitor. $J$ Neurosci 38:8822-8830.

Choi BR, Kim DH, Gallagher M, Han JS (2020) Engagement of the lateral habenula in the association of a conditioned stimulus with the absence of an unconditioned stimulus. Neuroscience 444:136148.

Chow JJ, Smith AP, Wilson AG, Zentall TR, Beckmann JS (2017) Suboptimal choice in rats: incentive salience attribution promotes maladaptive decision-making. Behav Brain Res 320:244-254.

Cohen JY, Amoroso MW, Uchida N (2015) Serotonergic neurons signal reward and punishment on multiple timescales. Elife 4:e06346.

Collins AGE, Frank MJ (2014) Opponent actor learning (OpAL): modeling interactive effects of striatal dopamine on reinforcement learning and choice incentive. Psychol Rev 121:337-366.

Cools R, Nakamura K, Daw ND (2011) Serotonin and dopamine: unifying affective, activational, and decision functions. Neuropsychopharmacology 36:98-113.

Couvillon PA, Bumanglag AV, Bitterman ME (2003) Inhibitory conditioning in honeybees. Q J Exp Psychol B 56:359-370.

Daly HB, Daly JT (1982) A mathematical model of reward and aversive nonreward: its application in over 30 appetitive learning situations. J Exp Psychol Gen 111:441-480.

Donaire R, Morón I, Blanco S, Villatoro A, Gámiz F, Papini MR, Torres C (2019) Lateral habenula lesions disrupt appetitive extinction, but do not affect voluntary alcohol consumption. Neurosci Lett 703:184-190.

Durrieu M, Wystrach A, Arrufat P, Giurfa M, Isabel G (2020) Fruit flies can learn non-elemental olfactory discriminations. Proc Biol Sci 287:20201234.

Eder AB, Rothermund K, De Houwer J, Hommel B (2015) Directive and incentive functions of affective action consequences: an ideomotor approach. Psychol Res 79:630-649.

Frank MJ (2005) Dynamic dopamine modulation in the basal ganglia: A neurocomputational account of cognitive deficits in medicated and nonmedicated Parkinsonism. J Cog Neuro 17:51-72.

Felsenberg J, Barnstedt O, Cognigni P, Lin S, Waddell S (2017) Reevaluation of learned information in Drosophila. Nature 544:240244.

Gold PW, Kadriu B (2019) A major role for the lateral habenula in depressive illness: physiologic and molecular mechanisms. Front Psychiatry 10:1-7.

Haddon JE, Killcross S (2011) Inactivation of the infralimbic prefrontal cortex in rats reduces the influence of inappropriate habitual responding in a response-conflict task. Neuroscience 199:205-212.

Hardung S, Epple R, Jäckel Z, Eriksson D, Uran C, Senn V, Gibor L, Yizhar O, Diester I (2017) A functional gradient in the rodent prefrontal cortex supports behavioral inhibition. Curr Biol 27:549-555.
Harris JA (2019) The importance of trials. J Exp Psychol Anim Learn Cogn 45:390-404.

Harris JA, Kwok DWS, Andrew BJ (2014) Conditioned inhibition and reinforcement rate. J Exp Psychol Anim Learn Cogn 40:335-354.

Hengartner MP, Davies J, Read J (2019) Antidepressant withdrawal the tide is finally turning. Epidemiol Psychiatr Sci 29:e52.

Hikosaka O (2010) The habenula: from stress evasion to value-based decision-making. Nat Rev Neurosci 11:503-513.

Hong S, Hikosaka O (2008) The globus pallidus sends reward-related signals to the lateral habenula. Neuron 60:720-729.

Hong S, Hikosaka O (2013) Diverse sources of reward value signals in the basal ganglia nuclei transmitted to the lateral habenula in the monkey. Front Hum Neurosci 7:1-7.

Hong S, Amemori S, Chung E, Gibson DJ, Amemori K, Graybiel AM (2019) Predominant striatal input to the lateral habenula in macaques comes from striosomes. Curr Biol 29:51-61.e5.

$\mathrm{Hu} \mathrm{H}$, Cui Y, Yang Y (2020) Circuits and functions of the lateral habenula in health and in disease. Nat Rev Neurosci 21:277-295.

Huang S, Borgland SL, Zamponi GW (2019) Dopaminergic modulation of pain signals in the medial prefrontal cortex: challenges and perspectives. Neurosci Lett 702:71-76.

lordanova MD, Yau JOY, McDannald MA, Corbit LH (2021) Neural substrates of appetitive and aversive prediction error. Neurosci Biobehav Rev 123:337-351.

Jakobs M, Pitzer C, Sartorius A, Unterberg A, Kiening K (2019) Acute $5 \mathrm{~Hz}$ deep brain stimulation of the lateral habenula is associated with depressive-like behavior in male wild-type Wistar rats. Brain Res 1721:146283.

Jhou TC, Geisler S, Marinelli M, Degarmo BA, Zahm DS (2009) The mesopontine rostromedial tegmental nucleus: a structure targeted by the lateral habenula that projects to the ventral tegmental area of Tsai and substantia nigra compacta. J Comp Neurol 513:566596.

Jo YS, Lee J, Mizumori SJY (2013) Effects of prefrontal cortical inactivation on neural activity in the ventral tegmental area. J Neurosci 33:8159-8171.

Juarez J, Barrios De Tomasi E, Muñoz-Villegas P, Buenrostro M (2013) Adicción farmacológica y conductual. In: Cerebro y conducta (González-Garrido A, and Matute E, eds). Guadalajara: Manual Moderno.

Konorski J (1948) Conditioned reflexes and neuron organization. New York: Cambridge University Press.

Lammel S, Lim BK, Ran C, Huang KW, Betley MJ, Tye KM, Deisseroth K, Malenka RC (2012) Input-specific control of reward and aversion in the ventral tegmental area. Nature 491:212-217.

Laubach M, Caetano MS, Narayanan NS (2015) Mistakes were made: neural mechanisms for the adaptive control of action initiation by the medial prefrontal cortex. J Physiol Paris 109:104-117.

Laubach M, Amarante LM, Swanson K, White SR (2018) What, if anything, is rodent prefrontal cortex? eNeuro 5:ENEURO.031518.2018-333.

Laude JR, Stagner JP, Zentall TR (2014) Suboptimal choice by pigeons may result from the diminishing effect of nonreinforcement. J Exp Psychol Anim Learn Cogn 40:12-21.

Laurent V, Wong FL, Balleine BW (2017) The lateral habenula and its input to the rostromedial tegmental nucleus mediates outcomespecific conditioned inhibition. J Neurosci 37:10932-10942.

Lecourtier L, DeFrancesco A, Moghaddam B (2008) Differential tonic influence of lateral habenula on prefrontal cortex and nucleus accumbens dopamine release. Eur J Neurosci 27:1755-1762.

Li H, Vento PJ, Parrilla-Carrero J, Pullmann D, Chao YS, Eid M, Jhou TC (2019) Three rostromedial tegmental afferents drive triply dissociable aspects of punishment learning and aversive valence encoding. Neuron 104:987-999.e4.

Mathis V, Barbelivien A, Majchrzak M, Mathis C, Cassel JC, Lecourtier $L$ (2017) The lateral habenula as a relay of cortical information to process working memory. Cereb Cortex 27:5485-5495.

Matthews GA, Nieh EH, Vander Weele CM, Halbert SA, Pradhan RV, Yosafat AS, Glober GF, Izadmehr EM, Thomas RE, Lacy GD, Wildes CP, Ungless MA, Tye KM (2016) Dorsal raphe dopamine 
neurons represent the experience of social isolation. Cell 164:617631.

Matsumoto M, Hikosaka O (2007) Lateral habenula as a source of negative reward signals in dopamine neurons. Nature 447:11111115.

Meyer HC, Bucci DJ (2014) The contribution of medial prefrontal cortical regions to conditioned inhibition. Behav Neurosci 128:644-653.

Mollick JA, Hazy TE, Krueger KA, Nair A, Mackie P, Herd SA, O'Reilly RC (2020) A systems-neuroscience model of phasic dopamine. Psychol Rev 127:972-1021.

Mollick JA, Chang LJ, Krishnan A, Hazy TE, Krueger KA, Frank GKW (2021) The neural correlates of cued reward omission. Front Hum Neurosci 15:615313.

O’Reilly RC, Russin J, Herd SA (2019) Computational models of motivated frontal function. Hand Clin Neurol 163:317-332.

Omelchenko N, Sesack SR (2009) Ultrastructural analysis of local collaterals of rat ventral tegmental area neurons: GABA phenotype and synapses onto dopamine and GABA cells. Synapse 63:895906.

Ongur D, Price JL (2000) The organization of networks within the orbital and medial prefrontal cortex of rats, monkeys and humans. Cereb Cortex 10:206-219.

Papalini S, Beckers T, Vervliet B (2020) Dopamine: from prediction error to psychotherapy. Transl Psychiatry 10:164.

Papini MR, Bitterman ME (1993) The two-test strategy in the study of inhibitory conditioning. J Exp Psychol Anim Behav Process 19:342-352.

Papini MR, Wood M, Daniel AM, Norris JN (2006) Reward loss as psychological pain. Int J Psychol Psychol Ther 6:189-213.

Pearce JM, Hall G (1980) A model for Pavlovian learning: variations in the effectiveness of conditioned but not of unconditioned stimuli. Psychol Rev 87:532-552.

Perisse E, Owald D, Barnstedt O, Talbot CBB, Huetteroth W, Waddell S (2016) Aversive learning and appetitive motivation toggle feed-forward inhibition in the Drosophila mushroom body. Neuron 90:1086-1099.

Piantadosi PT, Yeates DCM, Floresco SB (2020) Prefrontal cortical and nucleus accumbens contributions to discriminative conditioned suppression of reward-seeking. Learn Mem 27:429-440.

Proulx CD, Hikosaka O, Malinow R (2014) Reward processing by the lateral habenula in normal and depressive behaviors. Nat Neurosci 17:1146-1152.

Ragozzino ME, Detrick S, Kesner RP (1999) Involvement of the prelimbic-infralimbic areas of the rodent prefrontal cortex in behavioral flexibility for place and response learning. $J$ Neurosci 19:4585-4594.

Rescorla RA (1969) Pavlovian conditioned inhibition. Psychol Bull 72:77-94.

Rescorla R, Wagner AR (1972) A theory of classical conditioning: variations in the effectiveness of reinforcement and nonreinforcement. In: Classical conditioning II: current research and theory. New York: Appleton-Century-Crofts.

Roesch MR, Esber GR, Li J, Daw ND, Schoenbaum G (2012) Surprise! Neural correlates of Pearce-Hall and Rescorla-Wagner coexist within the brain. Eur J Neurosci 35:1190-1200.

Roughley S, Killcross S (2019) Loss of hierarchical control by occasion setters following lesions of the prelimbic and infralimbic medial prefrontal cortex in rats. Brain Sci 9:48-16.

Salas R, Baldwin P, de Biasi M, Montague PR (2010) BOLD responses to negative reward prediction errors in human habenula. Front Hum Neurosci 4:1-7.

Santini E, Quirk GJ, Porter JT (2008) Fear conditioning and extinction differentially modify the intrinsic excitability of infralimbic neurons. J Neurosci 28:4028-4036.

Savastano HI, Cole RP, Barnet RC, Miller RR (1999) Reconsidering conditioned inhibition. Learn Motiv 30:101-127.

Schmidt ERE, Pasterkamp RJ (2017) The molecular mechanisms controlling morphogenesis and wiring of the habenula. Pharmacol Biochem Behav 162:29-37.
Schultz W, Dayan P, Montague PR (1997) A neural substrate of prediction and reward. Science 275:1593-1599.

Sharpe MJ, Killcross S (2015) The prelimbic cortex directs attention toward predictive cues during fear learning. Learn Mem 22:289293.

Sharpe MJ, Killcross S (2018) Modulation of attention and action in the medial prefrontal cortex of rats. Psychol Rev 125:822-843.

Sosa R, dos Santos CV (2019a) Toward a unifying account of impulsivity and the development of self-control. Perspect Behav Sci 42:291-322.

Sosa R, dos Santos CV (2019b) Conditioned inhibition and its relationship to impulsivity: empirical and theoretical considerations. Psychol Rec 69:315-332.

Sosa R, Ramírez MN (2019) Conditioned inhibition: historical critiques and controversies in the light of recent advances. J Exp Psychol Anim Learn Cogn 45:17-42.

Sotres-Bayon F, Sierra-Mercado D, Pardilla-Delgado E, Quirk GJ (2012) Gating of fear in prelimbic cortex by hippocampal and amygdala inputs. Neuron 76:804-812

Stamatakis AM, Van Swieten M, Basiri ML, Blair GA, Kantak P, Stuber GD (2016) Lateral hypothalamic area glutamatergic neurons and their projections to the lateral habenula regulate feeding and reward. J Neurosci 36:302-311.

Stephenson-Jones M, Bravo-Rivera C, Ahrens S, Furlan A, Xiao X, Fernandes-Henriques C, Li B (2020) Opposing contributions of GABAergic and glutamatergic ventral pallidal neurons to motivational behaviors. Neuron 105:921-933.e5.

Stopper CM, Floresco SB (2014) What's better for me? Fundamental role for lateral habenula in promoting subjective decision biases. Nat Neurosci 17:33-35.

Sutton RS, Barto AG (2018) Reinforcement learning: an introduction, Ed 2. Cambridge: The MIT Press.

Tchenio A, Lecca S, Valentinova K, Mameli M (2017) Limiting habenular hyperactivity ameliorates maternal separation-driven depressive-like symptoms. Nat Commun 8:e01192-1.

Tian J, Uchida N (2015) Habenula lesions reveal that multiple mechanisms underlie dopamine prediction errors. Neuron 87:1304-1316.

Tian J, Huang R, Cohen JY, Osakada F, Kobak D, Machens CK, Callaway EM, Uchida N, Watabe-Uchida M (2016) Distributed and mixed information in monosynaptic inputs to dopamine neurons. Neuron 91:1374-1389.

Tobler PN, Dickinson A, Schultz W (2003) Coding of predicted reward omission by dopamine neurons in a conditioned inhibition paradigm. J Neurosci 23:10402-10410.

Tsutsui-Kimura I, Matsumoto H, Uchida N, Watabe-Uchida M (2020) Distinct temporal difference error signals in dopamine axons in three regions of the striatum in a decision-making task. bioRxiv. doi: 10.1101/2020.08.22.262972.

van Wijk BCM, Alkemade A, Forstmann BU (2020) Functional segregation and integration within the human subthalamic nucleus from a micro- and meso-level perspective. Cortex 131:103-113.

Vander Weele CM, Siciliano CA, Matthews GA, Namburi P, Izadmehr EM, Espinel IC, Nieh EH, Schut EHS, Padilla-Coreano N, BurgosRobles A, Chang C-J, Kimchi EY, Beyeler A, Wichmann R, Wildes CP, Tye KM (2018) Dopamine enhances signal-to-noise ratio in cortical-brainstem encoding of aversive stimuli. Nature 563:397401.

Varga V, Kocsis B, Sharp T (2003) Electrophysiological evidence for convergence of inputs from the medial prefrontal cortex and lateral habenula on single neurons in the dorsal raphe nucleus. Eur $\mathrm{J}$ Neurosci 17:280-286.

Vento PJ, Jhou TC (2020) Bidirectional valence encoding in the ventral pallidum. Neuron 105:766-768.

Verbruggen F, Best M, Bowditch WA, Stevens T, McLaren IPL (2014) The inhibitory control reflex. Neuropsychologia 65:263-278.

Vitay J, Hamker FH (2014) Timing and expectation of reward: a neuro-computational model of the afferents to the ventral tegmental area. Front Neurorobot 8:1-25. 
Review 16 of 16

Vogel EH, Ponce FP, Wagner AR (2019) The development and presint status of the SOP model of associative learning. Q J Exp Psychol (Hove) 72:346-374.

Wagner AR, Rescorla RA (1972) Inhibition in Pavlovian conditioning: application of a theory. In: Inhibition and learning, pp 301-334. New York: Academic Press.

Wang D, Li Y, Fens Q, Guo Q, Chou J, Lu M (2017) Learning shapes the aversion and reward responses of lateral habenula neurons. Elife 6:e23045.

Wasserman EA, Franklin SR, Hearst E (1974) Pavlovian appetitive contingencies and approach versus withdrawal to conditioned stimuli in pigeons. J Comp Physio Psychol 86:616-627.

Wheeler RA, Corelli RM (2006) The neuroscience of pleasure. Focus on ventral pallidum firing codes hedonic reward: when a bad taste turns good. J Neurophysiol 96:2175-2176.

Wiecki TV, Frank MJ (2013) A computational model of inhibitory control in frontal cortex and basal ganglia. Psychol Rev 120:329-355.

Yagishita S, Hayashi-Takagi A, Ellis-Davies GCR, Urakubo H, Ishii S, Kasai H (2014) A critical time window for dopamine actions on the structural plasticity of dendritic spines. Science 345:1616-1620.

Yon R, Wang T, Chou Q (2019) Elevated dopamine signaling from ventral tegmental area to prefrontal cortical parvalbumin neurons drives conditioned inhibition. Proc Natl Aced Sci USA 116:1307713086.

Yetnikoff L, Cheng AY, Lavezzi HN, Parsley KP, Zahm DS (2015) Sources of input to the rostromedial tegmental nucleus, ventral tegmental area, and lateral habenula compared: a study in rat. $J$ Comp Neurol 523:2426-2456.

Yin $H$ (2020) The crisis in neuroscience. In: The interdisciplinary handbook of perceptual control theory. Amsterdam: Elsevier Inc.

Zapata A, Hwang EK, Lupica CR (2017) Lateral habenula involvemont in impulsive cocaine seeking. Neuropsychopharmacology 42:1103-1112.

Thu Y, Qi S, Chang B, He D, Tens Y, Mu J, Wei H (2019) Connectome-based biomarkers predict subclinical depression and identify abnormal brain connections with the lateral habenula and thalamus. Front Psychiatry 10:1-14.

May/June 2021, 8(3) ENEURO.0016-21.2021

eNeuro.org 